\title{
Implicaciones de la criminalización del aborto en Ecuador
}

\author{
Elsa Guerra Rodríguez*
}

\begin{abstract}
El aborto libre como opción de las mujeres, ha venido siendo un recurso para dignificar la vida humana. El aborto libre como opción de las mujeres, es un recurso fundamental, entre otros, para recrear la maternidad, banalizada como trabajo propiamente animal, "descualificado", a la vez que sometida a normas de crianza autoritaria. No es solo un asunto de derechos individuales de las mujeres, sin los que es imposible apoyar el desarrollo pleno de una vida humana, sino también de derechos comunes; es un recurso necesario para la gestión de una vida digna en la diversidad.
\end{abstract}

Andrea Aguirre

\begin{abstract}
RESUMEN
El artículo evidencia el carácter ineficaz y anacrónico de la penalización del aborto, ya que, además de generar graves violaciones a los derechos de las mujeres, ha provocado que los índices de morbimortalidad femenina continúen creciendo, y que los núcleos familiares, la sociedad y el Estado sean los afectados. Adicionalmente, visibiliza la manera en que la criminalización de la interrupción del embarazo se configura en un dispositivo de control del cuerpo femenino y de exigencia de la observancia de un contrato reproductivo impuesto por el sistema patriarcal. Finalmente, plantea algunas alternativas frente a la necesidad de reducir los índices alarmantes del aborto clandestino en Ecuador, y de la demanda feminista de reconocimiento de las mujeres como sujetos plenos y capaces de autodeterminarse en el campo reproductivo.

Palabras Clave: criminalización del aborto, mujeres, género, interseccionalidad, derechos constitucionales, feminismos, patriarcado.
\end{abstract}

\section{ABSTRACT}

The article demonstrates the ineffective and anachronistic nature of the criminalization of abortion, since in addition to generating serious violations of women's rights, it has caused that the female morbidity and mortality rates continue growing,

* Docente de planta de la Universidad Andina Simón Bolívar, Sede Ecuador. 
and that the family nucleus, society and the State are the affected. Additionally, it makes visible the way in which the criminalization of the interruption of pregnancy is configured as a device for the control of the female body and the requirement of the observance of a reproductive contract imposed by the patriarchal system. Finally, it raises some alternatives to the need to reduce the alarming rates of clandestine abortion in Ecuador, and the feminist demand for recognition of women as full subjects capable of self-determination in the reproductive field.

KEYWORDS: criminalization of abortion, women, gender, intersectionality, constitutional rights, feminism, patriarchy.

\section{INTRODUCCIÓN}

$\mathrm{R}$ esulta paradójico que, mientras el marco constitucional ecuatoriano mediante varios de sus enunciados normativos prohíbe algunas de las formas de opresión y discriminación, la vigencia de normas infraconstitucionales, como la penalización de la interrupción del embarazo, se instituye en uno de los mecanismos que restringe de forma irrazonable el ejercicio de los derechos de las mujeres diversas.

En este sentido, el presente artículo busca evidenciar el carácter anacrónico de la penalización del aborto y la necesidad de bosquejar nuevas alternativas que, además de replantear esta realidad social ecuatoriana, cristalicen la posibilidad de consolidar una mirada transformadora del derecho y de la sociedad, que observe a la mujer como un fin en sí misma.

Para cumplir con este objetivo, este documento se divide en tres subtemas. E1 primero analizará la incidencia del aborto clandestino e inseguro en Ecuador como un problema de salud pública que afecta la integridad personal y vida de las mujeres que deciden abortar, la sociedad y el Estado; el segundo evidenciará la forma en que estas afectaciones provocadas por la penalización de la interrupción del embarazo se traducen en la afectación de un sinnúmero de derechos constitucionales y humanos y la forma cómo su tipificación no cumple eficazmente con la protección de la potencial vida del no nato; y el tercero examinará las implicancias simbólicas articuladas de la manera en que la criminalización del aborto voluntario constituye una herramienta patriarcal para instrumentalizar el cuerpo de las mujeres diversas. 


\title{
IMPLICANCIAS OBJETIVAS Y SIMBÓLICAS DE LA CRIMINALIZACIÓN DEL ABORTO EN ECUADOR
}

\author{
LAS AFECTADAS POR LA PENALIZACIÓN DEL ABORTO \\ Y SU PRÁCTICA CLANDESTINA: LAS MUJERES DIVERSAS, \\ LA SOCIEDAD Y EL ESTADO
}

El aborto es la segunda práctica ginecológica más habitual. En América Latina su incidencia es alarmante. Por ejemplo, en Brasil, se ejecutan un millón de interrupciones de embarazos anualmente. En Perú de cada 7 mujeres 1 aborta. $^{1}$

En la realidad ecuatoriana, la práctica del aborto clandestino e inseguro es aún más grave. Según CLACAI, Ecuador es el país donde se practican con mayor frecuencia abortos en Latinoamérica. ${ }^{2}$ Alrededor de 95.000 mujeres interrumpen sus embarazos anualmente. Frente a este índice, el Instituto Nacional de Estadística y Censos (INEC) ha indicado que cada año se realizan 200 abortos legales, ${ }^{3}$ circunstancia que implica que el resto de abortos se practica de forma oculta y, consecuentemente, con un alto grado de peligrosidad para la protección de la integridad personal y vida de las mujeres. $^{4}$

Estos índices como efecto de la criminalización del aborto voluntario han generado afectaciones graves e irreparables en la vida e integridad de las mujeres, en los núcleos familiares, en la sociedad y en el mismo Estado.

1. Comisión Interamericana de Derechos Humanos, Audiencia temática: criminalización del aborto en Sudamérica, 15 de marzo de 2006.

2. Colectivo Político Luna Creciente, Estado de arte de derechos sexuales y derechos reproductivos de las mujeres en el Ecuador (Quito: Fondo de Cooperación al Desarrollo de Solidaridad Socialista Belga, 2013), 28-9.

3. Sara Larrea, Diagnóstico de la situación de la promoción, oferta y demanda de la anticoncepción de emergencia en Loja, Guayas, Pichincha, Esmeraldas y Chimborazo (Quito: Fundación Desafío y Coordinadora Juvenil por la Equidad de Género, 2010), 15.

4. El Código Orgánico Integral Penal señala: “Artículo 149. Aborto consentido. La persona que haga abortar a una mujer que ha consentido en ello, será sancionada con pena privativa de libertad de uno a tres años. La mujer que cause su aborto o permita que otro se lo cause, será sancionada con pena privativa de libertad de seis meses a dos años.

Artículo 150. Aborto no punible. El aborto practicado por un médico u otro profesional de la salud capacitado, que cuente con el consentimiento de la mujer o de su cónyuge, pareja, familiares íntimos o su representante legal, cuando ella no se encuentre en posibilidad de prestarlo, no será punible en los siguientes casos: 1 . Si se ha practicado para evitar un peligro para la vida o salud de la mujer embarazada y si este peligro no puede ser evitado por otros medios. 2. Si el embarazo es consecuencia de una violación en una mujer que padezca de discapacidad mental". 
Respecto a la vida e integridad femenina, cabe mencionar que las mujeres conminadas por diversas motivaciones recurren a la práctica de abortos clandestinos, circunstancia que en la mayoría de los casos genera afectaciones a su vida e integridad personal. Frente a la mortalidad materna, la Organización Mundial de la Salud ha señalado que el $13 \%$ de las muertes maternas a nivel mundial se producen por causa de abortos en condiciones inseguras. En Latinoamérica y el Caribe, alrededor de 5.000 mujeres fallecen por esta práctica en circunstancias de clandestinidad. ${ }^{5}$ Según ENDEMAIN, el aborto en Ecuador es la segunda causa de muertes maternas, provocadas en su mayoría por hemorragias. ${ }^{6}$

En el caso de la integridad de la mujer, esta sufre afectaciones físicas y psicológicas, siendo la interrupción del embarazo la primera causa de morbilidad materna en Ecuador. Esta afirmación implica que un alto porcentaje de mujeres se someten a tratamientos de prorrogada duración para su recuperación, y otro grupo soporta daños permanentes por esta práctica en circunstancias inseguras. ${ }^{7}$

Además de las afectaciones físicas, se evidencian daños psico-emocionales generados por el proceso de culpa que se agudizan a partir del rechazo social como medida de sanción moral impuesta en sociedades patriarcales como la ecuatoriana:

En el caso de la Maternidad Isidro Ayora de la ciudad de Quito, por ejemplo, se reportó que a pesar de que existen mecanismos más eficaces, económicos, menos dolorosos y sencillos para retirar restos ovulares en caso de abortos incompletos, como la Aspiración Manual Endouterina (AMEU), todavía se ejecutan los legrados tradicionales, cuyo procedimiento es más complejo, doloroso y requiere mayor tiempo de recuperación. Esta condición permite entrever la forma en que el personal hospitalario reprime física y moralmente el comportamiento de la mujer al decidir abortar, situación que se reproduce en otros centros hospitalarios del Ecuador. ${ }^{8}$

Finalmente, además de ser víctimas de aislamiento y prejuicio como un mecanismo de castigo, las mujeres recurren en algunos casos al suicidio como forma de huir de este proceso de exclusión y muerte social. ${ }^{9}$

5. Coordinadora Política Juvenil por la Equidad de Género, ;De eso no se habla! (Quito: CPJ), 13.

6. Virginia Gómez de la Torre, "Derechos económicos, sociales y culturales. La salud de las mujeres al 2010 en el Ecuador", en Informe Derechos Humanos Ecuador 2009-2010 (Quito: Centro Regional de Derechos Humanos y Justicia de Género, 2011), 118.

7. Ver sobre las afectaciones físicas de las mujeres provocadas por la práctica del aborto clandestino en Aníbal Faúndes y José Barzelatto, El drama del aborto. En busca de consensos (Buenos Aires: Paidós, 2011).

8. Elsa Guerra, "La constitucionalidad del aborto voluntario en Ecuador" (tesis de maestría, Universidad Andina Simón Bolívar, Sede Ecuador, 2013), 32. Ver María Rosa Cevallos, El temor encarnado (Quito: FLACSO Ecuador, 2012).

9. Ver Virginia Gómez y Marta López, Decisiones Cotidianas (Quito: Fundación Desafío/Hivos, 2011), 53. 
En el caso de las afectaciones en los núcleos familiares y en la sociedad por la ilegalidad del aborto, cabe mencionar que el menoscabo a la vida e integridad femenina, a causa de una interrupción del embarazo practicada en circunstancias inadecuadas, también produce efectos negativos en la familia y en la sociedad. Esta afirmación tiene asidero en el rol fundamental que cumplen las mujeres tanto dentro como fuera del hogar, no solo por su capacidad reproductiva sino productiva. Según CLADEM, "las estadísticas nacionales muestran que las mujeres tienen [...] mayor carga global de trabajo que los hombres (9 horas), dedican 20 horas más en promedio por semana al trabajo doméstico y son responsables del $75 \%$ del tiempo dedicado al cuidado de niños-as". ${ }^{10}$

Estas cifras evidencian el papel que desempeñan las mujeres, sobre todo en países como Ecuador donde todavía existe un impacto de la división de roles de género, circunstancia que implica que, además de la carga laboral que cumplen fuera de casa, son el eje angular del cuidado de los niños, de las tareas domésticas, entre otras actividades. ${ }^{11}$

Respecto a las secuelas del aborto clandestino frente al Estado, en Ecuador, a inicios del año 2000, "los egresos hospitalarios del país por aborto representaron el $4 \%$, ocupando el primer lugar de la morbilidad femenina, excluyendo la atención de los partos. En 2008, la situación no varió en términos porcentuales, representando el $3,6 \% "{ }^{12}$ Por lo tanto, además de la pérdida de fuerza de trabajo dentro y/o fuera de la esfera privada del hogar, el Estado gasta en la recuperación de las afectaciones que viven las mujeres que interrumpieron su embarazo en circunstancias clandestinas e inseguras.

Finalmente, se evidencia una condición de interseccionalidad ${ }^{13}$ en el caso del aborto clandestino, por cuanto su ilegalidad aqueja con distinta intensidad a las mujeres de acuerdo a sus diversas subjetividades, rasgos distintivos y condiciones de vida

10. Comité de América Latina y el Caribe para la Defensa de los Derechos de la Mujer (CLADEM), investigación 2009.

11. Además se calcula que se ha perdido un aproximado de 1'800.000 años de la vida productiva de las mujeres a causa del aborto clandestino a nivel mundial, de los cuales el $98 \%$ pertenecen a las mujeres que residen en Estados subdesarrollados. Henry Espinoza y Lizbeth López, "Aborto inseguro en América Latina y el Caribe: definición del problema y su prevención", Gaceta Médica de México, vol. 139, suplem. 1 (julio-agosto 2003): 12. Disponible en 〈http://www.medigraphic.com/pdfs/gaceta/gm-2003/gms031c. pdf).

12. De la Torre, "Derechos económicos, sociales y culturales. La salud de las mujeres al 2010 en el Ecuador", 118.

13. Ver la explicación de este concepto en Alejandra de Lama Ayma, Discriminación múltiple I (Barcelona: Universidad Autónoma de Barcelona/Organización de las Naciones Unidas/Comité de la CEDAW), Recomendación No. 28. 
específicas en un tiempo y espacio determinado. ${ }^{14}$ Por ejemplo, el aborto efectuado en clínicas y con acompañamiento médico se ha convertido en un negocio rentable para cierta parte del mercado, e inaccesible para las mujeres pobres, constituyéndose este hecho social en un problema de clase.

Esta circunstancia ha obligado a que las mujeres de condiciones económicas limitadas históricamente busquen otras alternativas para interrumpir su embarazo, tales como el uso de alambres, sondas, palillos, detergentes, vinagre, raíces, ácidos, dosis inadecuadas de pastillas anticonceptivas, entre otros métodos. ${ }^{15}$

Respecto a las adolescentes, estas reciben con especial incidencia los efectos de la penalización del aborto consentido, ya que sufren una restricción a su autodeterminación atada a un doble proceso de imposición, primero, por parte del carácter adultocéntrico de la sociedad que, al observar a la juventud como un grupo asexuado e incapaz de decidir sobre su cuerpo de forma responsable, limita el acceso efectivo al uso de métodos anticonceptivos, ${ }^{16} \mathrm{y}$, segundo, frente a una cultura patriarcal que apoyada en la penalización del aborto voluntario ha provocado que "de las hospitalizaciones por abortos clandestinos, entre el 10 y el $21 \%$ [sean] adolescentes", ${ }^{17}$ y que 1 de cada 10 muertes maternas sea una adolescente. ${ }^{18}$

Adicionalmente, esta imposición ha logrado que un alto porcentaje de embarazos no deseados pertenezca a la población de niñas y adolescentes:

Según un estudio de la Organización Mundial de la Salud (OMS), [...] cada año se producen en el mundo 2 millones de partos de niñas menores de 15 años. De acuerdo a este estudio, América Latina y el Caribe es la única región donde los partos de niñas de menos de 15 años aumentaron, y se prevé que sigan aumentando al menos hasta el 2030. De acuerdo

14. En el caso de las mujeres indígenas y afroecuatorianas, el aparato estatal debería, generando un proceso de respeto a la cosmovisión de estos pueblos y a la decisión clara de la mujer frente a la intervención o no del Estado, asegurar el acceso a una atención médica oportuna que posibilite, por un lado, el uso de métodos anticonceptivos que es casi nulo (cinco veces menor que en la población mestiza), y viabilice la protección de la vida y salud de las mujeres antes, en el proceso y después de la interrupción de un embarazo. Ver Encuesta Demográfica y de Salud Materna e Infantil del Ecuador ENDEMAIN, 2004, 31.

15. Tomas Frejka, Lucille Atkin y Olga Toro, Programa de investigación para la prevención del aborto inducido en condiciones riesgosas y sus consecuencias adversas en América Latina y el Caribe (México: The Population Council, 1989), 18. Ver Radhira Coomaraswamy, "Informe de la Relatoría Especial sobre la violencia contra la mujer, sus causas y consecuencias", Revista Argentina de Derechos Humanos, Buenos Aires (2001): 365.

16. Ver Plan V, "Plan Familia Ecuador o un retroceso de cien años", Plan V, 14 de marzo de 2015.

17. Coordinadora Política Juvenil por la Equidad de Género, ¡De eso no se habla!, 14.

18. Ministerio de Salud Pública, Plan Nacional de Reducción Acelerada de la Mortalidad Materna y Neonatal, septiembre de 2008, 20. 
a este Organismo, en América Latina entre el 20\% (una de cada 5 mujeres) y el 9\% de las entrevistadas declaró haber sufrido abuso sexual en la infancia. ${ }^{19}$

Estos datos, además de demostrar al aborto clandestino como un hecho social cuya práctica es recurrente por encima de su criminalización, permite entrever la penalización de la interrupción del embarazo como aquella parte del derecho anacrónico que se construye sin mirar ni comprender la realidad, circunstancia que perjudica de forma significativa a las mujeres, a la sociedad y al Estado.

\section{LA CRIMINALIZACIÓN DEL ABORTO RESTRINGE IRRAZONABLEMENTE LOS DERECHOS CONSTITUCIONALES} Y HUMANOS DE LAS MUJERES

La Constitución ecuatoriana de 2008 incluye importantes avances respecto a la tutela de las mujeres diversas. Esta condición abarca la ampliación del contenido de varios derechos, y la garantía constitucional de la plena exigibilidad y justiciabilidad de los mismos, así como la obligatoriedad del establecimiento de medidas judiciales que tutelen a las mujeres y reparen integralmente sus derechos desde un enfoque de género e intercultural. ${ }^{20}$

Paralelamente, se instituye en una obligación de las instituciones públicas y privadas al observar las disposiciones contenidas en el derecho internacional de derechos humano ${ }^{21}$ que salvaguarden y garanticen la igualdad de género en la materialización de los derechos de las mujeres de cara al principio pro persona. ${ }^{22}$

Paradójicamente, aunque se resaltan estos destacados progresos en el campo jurídico, todas las afectaciones antes descritas provocadas por la penalización del aborto generan serias restricciones al ejercicio de los derechos constitucionales y humanos de las mujeres diversas, entre los que se destacan: el derecho a la vida, al libre de-

19. Inter-American Commission of Women, Informe hemisférico sobre violencia sexual y embarazo infantil en los Estados Parte de la Convención de Belém do Pará (Washington D. C.: OEA, 2016), 11.

20. A pesar de los avances normativos antes señalados, cierto contenido de la Constitución ecuatoriana de 2008 todavía responde a planteamientos estereotipados marcados por el patriarcado y otros sistemas de dominación. Por ejemplo, la imposibilidad de que dos personas del mismo sexo puedan adoptar a un niño o niña, o puedan contraer matrimonio, son una muestra del carácter discriminatorio y patriarcal que todavía se encuentra enraizado en sociedades como la ecuatoriana.

21. Ver Danilo Caicedo, "El bloque de constitucionalidad en el Ecuador. Derechos humanos más allá de la Constitución”, FORO: Revista de Derecho, No. 12, Universidad Andina Simón Bolívar, Sede Ecuador/ Corporación Editora Nacional (2009).

22. Ver Ximena Medellín, Principio Pro persona (México: Suprema Corte de Justicia de la Nación-Oficina en México del Alto Comisionado de las Naciones Unidas para los Derechos Humanos, 2013). 
sarrollo de la personalidad, a la salud, a la autonomía reproductiva, al principio de igualdad, la prohibición de discriminación, vida digna, intimidad, integridad personal, así como al principio de dignidad humana.

En el caso de la tutela a la salud, la Constitución ecuatoriana ha ampliado su contenido para observar este derecho desde su carácter integral, no solo desde su ámbito curativo sino preventivo, correlacionando particularmente su efectividad con la protección a la vida e integridad personal, y enfocando gran parte de su ejercicio en la cristalización de los derechos sexuales y reproductivos.

A pesar de este marco constitucional de protección, la penalización del aborto consentido restringe el acceso a una atención médica oportuna, y, en consecuencia, al ejercicio del derecho a una salud reproductiva. Por ello los organismos internacionales de protección de los derechos humanos han recomendado a los Estados que penalizan el aborto de forma total o parcial reformular su normativa con la finalidad de asegurar el acceso a la salud a las mujeres, mediante un servicio médico óptimo, y precautelar la cristalización de los derechos humanos. Además, han recalcado que la despenalización constituiría una forma de frenar los altos índices de abortos clandestinos e inseguros. ${ }^{23}$

Respecto al derecho a la vida e integridad personal de las mujeres, tanto la Constitución ecuatoriana como el derecho internacional de derechos humanos prohíben toda acción u omisión que cause afectaciones en la integridad física, psíquica y emocional de las personas; sin embargo, la penalización del aborto articulada al carácter moral de la sociedad patriarcal genera una vulneración irrazonable de este derecho, que provoca en numerosas ocasiones un menoscabo al derecho a la vida de las mujeres.

En el Caso Llantoy Huamán vs. Perú, el Comité de Derechos Humanos de la Organización de las Naciones Unidas fue claro en aseverar que "el derecho a la vida no puede entenderse de manera restrictiva, sino que de hecho requiere que los Estados adopten medidas positivas para su protección, incluyendo las medidas necesarias para evitar que las mujeres recurran a abortos clandestinos que pongan en peligro su salud y su vida especialmente cuando se trata de mujeres pobres". ${ }^{4}$

En este mismo contexto, este Comité estableció que la prohibición de tortura, tratos crueles, inhumanos o degradantes involucra, además del sufrimiento físico, aquel

23. El Plan Nacional del Buen Vivir 2013-2017 afirma que "en el caso de la tasa de mortalidad materna [...] no se ha logrado impactar en este indicador, que ha oscilado entre 48,5 y 69,7 entre 2001 y 2010". Secretaría Nacional de Planificación y Desarrollo del Ecuador, Plan Nacional del Buen Vivir 2013-2017 (Quito: SENPLADES), 137.

24. Organización de las Naciones Unidas, Dictamen del Comité de Derechos Humanos No. 1153/2003, 85 Período de Sesiones, 17 de noviembre de 2005. 
articulado a connotaciones emocionales, condición que implica que las manifestaciones de rechazo social y el sentimiento de culpa a causa de la ilegalidad de la interrupción voluntaria del embarazo transgreden la integridad psíquica y moral, provocando una especie de tortura y autoflagelación. ${ }^{25}$

Frente a este escenario, es necesario resaltar que la normativa constitucional e internacional permitiría derogar la penalización del aborto voluntario, ${ }^{26}$ que, articulada a los estereotipos marcados por el patriarcado, es contraria, entre otros derechos, al ejercicio de los derechos reproductivos, y a los estándares de protección de los derechos humanos de las mujeres como se procede a demostrar.

En el primer caso, la Constitución ecuatoriana estipula, como parte de los derechos reproductivos, la posibilidad de decidir cuándo y cuántos hijos tener. ${ }^{27}$ Este reconocimiento constitucional tiene consonancia con los estándares del Programa de Acción de la Conferencia Internacional sobre Población y Desarrollo del Cairo, así como con la Recomendación de la Organización Panamericana de la Salud que resalta, como parte de la materialización de una salud sexual y reproductiva, la posibilidad de que "las personas puedan disfrutar de una vida sexual satisfactoria, segura y responsable, así como la capacidad para reproducirse y la libertad de decidir si se reproducen, cuando y con qué frecuencia". ${ }^{28} \mathrm{El}$ ejercicio de este derecho, articulado a la cristalización de la igualdad material y el principio de dignidad humana, podría generar, como lo señala Ferrajoli, un derecho sexuado o "derecho-poder por así decir constituyente, de tipo pre- o meta-jurídico, puesto que es el reflejo de una potencia natural inherente de manera exclusiva a la diferencia femenina", ${ }^{29}$ que permita asegurar a las mujeres el acceso a una interrupción del embarazo en condiciones adecuadas.

En segundo lugar, a pesar de que la potencial vida del no nato es considerada como un fin legítimo de protección estatal, su estándar de resguardo se ha reducido frente al amparo de los derechos de las mujeres en el caso de la interrupción del embarazo.

En este sentido, la Corte Interamericana de Derechos Humanos, después de efectuar una interpretación histórica y sistemática de los antecedentes y trabajos preparatorios de la normativa del Sistema Interamericano, ha sostenido que ni la Convención Americana sobre Derechos Humanos, el Pacto Internacional de Derechos Civiles y Políticos, la Convención sobre los Derechos del Niño o la Declaración Universal de

25. Ibíd.

26. Es necesario aclarar que la despenalización no genera automáticamente una legalización de esta práctica.

27. Ecuador, Constitución de la República del Ecuador, Registro Oficial, No. 449, 20 de octubre de 2008, art. 66.

28. Organización Panamericana de la Salud, Salud en las Américas 2007, vol. I, Regional (Washington D. C.: OPS, 2007), 151.

29. Luigi Ferrajoli, Derechos y garantías: la ley del más débil, 2a. ed. (Madrid: Trotta, 1999), 85. 
los Derechos Humanos le otorgan el estatus de persona al embrión; $; 0$ por lo tanto, "las tendencias de regulación en el derecho internacional no llevan a la conclusión que el embrión sea tratado de manera igual a una persona o que tenga un derecho a la vida". ${ }^{31}$ Es decir que, aunque el producto de la concepción requiere una protección objetiva estatal, no es considerado una persona y, por lo tanto, no se le ha otorgado la capacidad plena de exigibilidad y justiciabilidad de derechos como sucede con la mujer haya o no interrumpido su embarazo.

Además, la Corte cuestiona la posibilidad de entender "el derecho a la vida como un derecho absoluto, cuya alegada protección pueda justificar la negación total de otros derechos", ${ }^{32}$ como sucede en el caso de la penalización del aborto, ya que esta tipificación provoca la restricción del ejercicio pleno de varios derechos constitucionales y humanos de las mujeres y genera graves afectaciones. Finalmente, este órgano reconoce que la protección a la vida del no nato debe ser gradual, y debe incrementarse de acuerdo al grado de viabilidad del no nacido. Esta interpretación incide en el peso que en la mayoría de países latinoamericanos se le ha otorgado tradicionalmente a la tutela de los derechos de las mujeres frente a la potencial vida del no nato, así como evidencia la necesidad de buscar nuevas alternativas que replanteen las consecuencias jurídicas y sociales del aborto clandestino e inseguro, tales como su despenalización, la legalización del mismo de acuerdo a leyes de plazo como han surgido en varios países, entre otras opciones. ${ }^{33}$

Finalmente, si bien la penalización de la interrupción voluntaria de la gestación ha sido generada con la finalidad de evitar cualquier menoscabo a la vida potencial desde la concepción, comprendido como un bien jurídico protegido por el Estado, ${ }^{34}$ la realidad del aborto clandestino evidencia su clara ineficacia frente al cumplimiento de esta obligación objetiva del aparato estatal, por cuanto el promedio anual de crecimiento de interrupciones de embarazo en el país en el período de 1998 a 2008 fue del $7 \% .^{35}$

30. Corte Interamericana de Derechos Humanos, Caso Artavia Murillo y Otros ("Fecundación in vitro") vs. Costa Rica, sentencia de 28 de noviembre de 2012.

31. Ibíd., párrafo 253.

32. Corte Interamericana de Derechos Humanos, Sentencia caso Artavia Murillo y otros ("Fecundación in vitro") vs. Costa Rica, sentencia de 28 de noviembre de 2012, párrafo 75.

33. En países como Cuba, México y Uruguay se ha reconocido la necesidad de legalizar el aborto voluntario dentro de las 12 primeras semanas de gestación, como mecanismo de solución de un conflicto que requiere ser visibilizado y resuelto, constituyéndose en un proceso rupturista en América Latina, que replantea la forma de analizar los derechos de libertad de la mujer respecto a su cuerpo, desarrollo, dignidad y su relación con el nonato.

34. Ecuador, Constitución de la República del Ecuador, Registro Oficial, No. 449, 20 de octubre de 2008, art. 45.

35. De la Torre, "Derechos económicos, sociales y culturales. La salud de las mujeres al 2010 en el Ecuador", 118. Cabe mencionar que el Tribunal Constitucional alemán en una de sus sentencias si bien declaró inconstitucional la legalización del aborto, sugirió a su poder legislativo que busque otros mecanismos 
Este dato reafirma lo señalado por Ramiro Ávila, quien sostiene que la tipificación del aborto como delito "ni es práctica porque no protege la vida intrauterina, ni es ética porque causa millones de muertes". ${ }^{36}$

Sin embargo, además de que esta práctica se ha acrecentado, dicha criminalización legal está acompañada de una penalización social, que genera una especie de muerte social para aquellas mujeres que han interrumpido su embarazo "sin razón justificada" desde un enfoque meramente patriarcal, incrementando los niveles de violencia contra ellas.

Consecuentemente, el Estado debería derogar la penalización de la interrupción del embarazo por cuanto es un impedimento para actuar de forma efectiva en la protección de los derechos constitucionales y bienes jurídicos, todos menoscabados por la criminalización de esta práctica, e indagar nuevos horizontes que permitan replantear la gravedad de este hecho social.

\section{LA PENALIZACIÓN DEL ABORTO ES UNA FORMA DE INSTRUMENTALIZAR}

\section{EL CUERPO DE LAS MUJERES DIVERSAS}

La criminalización de la interrupción del embarazo, además de permitir la violación de varios derechos constitucionales y humanos, también debe ser observada como una muestra del uso patriarcal del derecho para atar el cuerpo de las mujeres.

Sin duda, el patriarcado ha impuesto de acuerdo a la diferenciación sexual, comportamientos de lo masculino y femenino a hombres y mujeres, hegemonizando el primero sobre el segundo, estableciendo procesos de relegación y discriminación para lo femenino, ${ }^{37}$ y generando distintos dispositivos de control, a quienes se apartan de este orden binario. ${ }^{38}$

para proteger la vida del no nacido alejado de la penalización de la interrupción del embarazo. Ver Rafael Domingo, "El aborto en Alemania: (Observaciones sobre la sentencia del Tribunal Constitucional alemán de 28.5.1993)", Cuadernos de Bioética (1994): 213.

36. Alda Facio, "Metodología para el análisis de género del fenómeno legal", en Ramiro Ávila Santamaría, Judith Salgado y Lola Valladares, comp., El género en el derecho: Ensayos críticos (Quito: Ministerio de Justicia y Derechos Humanos, 2009), 190.

37. Lo femenino debe ser entendido como la categoría que engloba a todas aquellas personas que han adquirido comportamientos o generolectos de lo femenino, y que en la mayoría de los casos se reflejan en las características de las mujeres como elementos impuestos por el patriarcado, a partir de los cuales se generan procesos de opresión a quienes se muestren subversivos o subversivas a dicho a mandato.

38. Judith Salgado, Manual de formación en género y derechos humanos (Quito: Universidad Andina Simón Bolívar, Sede Ecuador/Corporación Editora Nacional, 2013). 
A partir de este vínculo de poder se han creado varios mecanismos de control e instrumentalización de las mujeres, entre ellos, el establecimiento de una dicotomía entre lo productivo-reproductivo, ${ }^{39}$ instaurando una división manifiesta de roles entre los géneros, condición que aseguró la construcción de un Estado y sociedad androcéntrica que excluía, explotaba y coartaba la autonomía de las mujeres y dotaba de exacerbado poder a la mayoría de hombres.

En este contexto, la exitosa estrategia del patriarcado ha sido interiorizarse en las relaciones sociales públicas, privadas, y socioestatales, de tal manera que ha naturalizado las distintas formas de relegación y violencia contra las mujeres, como parte del "pacto social".

Ahora bien, para evidenciar las relaciones asimétricas de poder entre los géneros, es necesario, como bien lo describe Joan Scott, analizar las tensiones creadas entre hombres y mujeres desde la dinámica simbólica, institucional, subjetiva y normativa, así como a partir de otras manifestaciones que aseguran la relegación de lo femenino. ${ }^{40}$

Respecto al campo normativo, es evidente que el derecho no ha sido neutral, ya que se ha constituido un dispositivo de dominación y exclusión, ${ }^{41}$ mediante el uso de diversos mecanismos tales como el androcentrismo, sobre generalización, dicotomismo sexual, sobre especificación, insensibilidad al género, doble parámetro, entre otros ${ }^{42}$ provocando que históricamente varias de las normas y su interpretación patriarcal invisibilicen lo femenino en el derecho, institucionalicen lo permitido y prohibido para las mujeres desde el pensamiento masculino, consoliden jurídicamente la división de roles sexuales y reproductivos para las mujeres, normalicen la doble o triple explotación de lo femenino frente a lo masculino dominante basada en su supuesta inferioridad, etcétera. ${ }^{43}$

En este sentido, si bien varios de los feminismos reconocen la incidencia del patriarcado y sexismo en el derecho, han establecido diferentes estrategias para erradi-

39. Catherine MacKinnon, Hacia una teoría feminista del Estado (Madrid: Cátedra, 1995), $23-4$.

40. Joan Scott, "El género una categoría útil para el análisis histórico", en Marta Lamas, comp., El género. La construcción cultural de la diferencia sexual (México: UNAM, 2003), 265-302.

41. La frase "no neutralidad del derecho" busca evidenciar la función ideológica del derecho ya que, más allá de la perspectiva formalista de sus fundamentos, impone mecanismos de opresión, subordinación y explotación para ciertos grupos humanos. Ver Katharine Bartlett, "Métodos jurídicos feministas”, en Marisol Fernández y Félix Morales, coord., Métodos feministas en el Derecho (Lima: Palestra, 2011), 19-116.

42. Facio, "Metodología para el análisis de género del fenómeno legal".

43. Estas normas pueden excluir a las mujeres, como sucedió en los inicios de nuestra República ecuatoriana cuando no se reconocía a la mujer como ciudadana y por lo tanto como sujeto de derechos; pueden instrumentalizar a la mujer, cuando el Estado no reconoce el trabajo del hogar como trabajo productivo y remunerado, o en el caso de la tipificación del aborto voluntario como delito en Ecuador y en otros países de América Latina. 
carlo y cristalizar los derechos de las mujeres diversas. ${ }^{44}$ En el caso de la autodeterminación reproductiva, se puede constatar que a lo largo de la historia han emergido corrientes feministas amplias y divergentes, entre las que se destacan por lo menos dos posiciones. La primera reivindica el reconocimiento por parte del Estado de facultades o derechos que permitan la tutela de este, en la cristalización de los derechos de libertad reproductiva de las mujeres, sobre todo como una necesidad emergente de aquellas personas que por su condición socioeconómica no pueden asegurar el ejercicio de estos derechos con sus propios recursos; y la segunda niega la posibilidad de que el Estado intervenga en el ejercicio de la autodeterminación reproductiva, ya que permitir su actuación involucraría aceptar una posible instrumentalización femenina y control estatal que históricamente ha servido de herramienta patriarcal. ${ }^{45}$

En este sentido, la penalización del aborto constituye una muestra clara del uso arbitrario del derecho para controlar el cuerpo de las mujeres, por cuanto es una forma de castigar el incumplimiento de un contrato sexual y reproductivo que determina maniobras biopolíticas, métodos discursivos y simbólicos que atan el comportamiento de las mujeres a los objetivos de la reproducción ${ }^{46} \mathrm{y} / \mathrm{o}$ a los fines ideológicos del patriarcado y el capitalismo, que pretenden categorizar a la identidad de la mujer con su potencial capacidad de ser madre, y a partir de ese rol exigen a esta cumplir con el proceso de reproducción de estos sistemas.

Ahora bien, cabe destacar que históricamente el no nato no siempre ha sido el bien jurídico protegido para penalizar el aborto; más bien han sido otros los fines que han encubierto el control de la autodeterminación sexual y reproductiva de las mujeres a lo largo de la historia.

Por ejemplo, "a pesar de que inicialmente en la época romana no se reconoció al aborto voluntario como un crimen, con posterioridad cuando la mujer casada de forma voluntaria interrumpía su embarazo se consideraba como delito por la afectación al marido sobre su derecho de descendencia". ${ }^{47}$ Finalmente, en Latinoamérica en el siglo XIX todavía se reconocía al aborto honoris causa como un atenuante o justificativo

44. Judith Salgado organiza a los Feminismos, a partir de los siguientes tipos: Feminismo de la igualdad, Feminismo liberales clásicos, Feminismo liberales sociales, Feminismo socialista, y Feminismo radical. Judith Salgado, "Aportes al debate sobre igualdad desde el feminismo", Revista Aportes Andinos, No. 9. Universidad Andina Simón Bolívar, Sede Ecuador (2004): 2.

45. Ver Catherine MacKinnon, Hacia una teoría feminista del Estado.

46. María Rosa Cevallos, El temor encarnado (Quito: FLACSO Ecuador, 2012), 20. Ver además, Tamar Pitch, Un derecho para dos. La construcción jurídica de género, sexo, sexualidad (Madrid: Trotta, 2003).

47. Guerra, "La constitucionalidad del aborto voluntario en Ecuador", 334. 
de la interrupción de la gestación siempre que busque proteger la reputación y honor de la mujer y/o de su familia. ${ }^{48}$

Estos argumentos demuestran que el discurso del paradigma garantista de los derechos queda obsoleto frente a una realidad que evidencia la vigencia del patriarcado como parte de las relaciones sociales, donde el derecho no es neutral, y más bien es parte de una estructura de dominación de lo femenino y de las mujeres diversas.

Más aún cuando en la actualidad el patriarcado continúa expresándose como fuente de dominación mediante procesos penales irregulares que criminalizan a mujeres por supuestos abortos ${ }^{49} \mathrm{y}$ sin observar un enfoque de género, se violenta varias normas del debido proceso, se persigue y vulnera su dignidad. ${ }^{50}$

\section{CONSIDERACIONES FINALES}

Es urgente vislumbrar otras medidas que logren tutelar la vida de las mujeres que, en su diversidad, decidan interrumpir sus embarazos, tomando en cuenta los estándares internacionales de protección de los derechos humanos, los límites al ejercicio de los derechos y las demandas reales de la sociedad, resaltando que no existen dispositivos de anticoncepción que aseguren la prevención efectiva de embarazos, circunstancia que visibiliza la complejidad de erradicar la práctica del aborto.

Gran parte de los feminismos y grupos sociales abogan, como alternativa eficaz a esta tipificación penal, la posibilidad real del acceso a un aborto seguro y gratuito, sobre todo para aquellas mujeres que por sus particulares condiciones no poseen recursos para utilizar métodos adecuados con un apoyo médico efectivo. Además insisten en que esta política debe ser encaminada mediante un proceso deliberativo que escuche las necesidades diversas de las mujeres. Sin embargo, cabe mencionar que el acceso a una interrupción del embarazo en condiciones apropiadas abre la posibilidad al Estado de intervenir nuevamente en el cuerpo femenino; por ello, es necesario bus-

48. Violeta Bermúdez, La regulación jurídica del aborto en América Latina y el Caribe. Estudio comparativo (Lima: CLADEM, 1998), 21-6.

49. Diego Bravo y Marcel Bonilla, "74 mujeres fueron enjuiciadas por abortar los dos últimos años", $E l$ Comercio, Actualidad, 25 de enero de 2016. Fecha de consulta: 12-01-2017. Disponible en 〈http://www. elcomercio.com/actualidad/ecuador-mujeres-juicios-aborto-carcel.html〉.

50. A contracorriente, las teorías feministas rescatan la connotación de la dignidad de las mujeres en el caso de la despenalización e inclusive legalización de la interrupción voluntaria del embarazo, por cuanto busca empoderar a las mujeres diversas como fines en sí mismas y no como un instrumento "o mero receptáculo", cuestionando la posibilidad de que, sacrificando su autodeterminación, se cumplan propósitos extraños a su voluntad, articulados a fines demográficos, o intereses del hombre o del Estado. Ver Colombia, Corte Constitucional de Colombia, Sentencia C-355 de 2006. 
car mecanismos que aseguren su participación razonable, acorde a la especial realidad de las mujeres y bajo una perspectiva de género.

Esta tarea debe estar acompañada por un proceso de despenalización social que deconstruya roles y estereotipos, y observe a las mujeres como personas con capacidad plena de gobernar sus cuerpos y proyectos de vida articulados a la reproducción, ${ }^{51}$ erradicando todo mecanismo de subordinación e instrumentalización.

Por otro lado, es indispensable recabar el aporte significativo de otros países que han legalizado la práctica de la interrupción del embarazo de acuerdo al grado de viabilidad del no nato, y que, además de reducir los índices de afectaciones a la vida e integridad de las mujeres por interrupciones clandestinas del embarazo, han logrado sembrar un amplio sentido de autodeterminación responsable del cuerpo, condición que ha permitido que se disminuya el índice de abortos. ${ }^{52}$

Finalmente, la lucha por replantear un derecho con rostro patriarcal evidenciado en normativas como la penalización del aborto, se enmarca en una batalla con un trasfondo más amplio y estructural, la equidad social. Por lo tanto, el reconocimiento y respeto a las diferencias y la promoción de garantías y de acciones afirmativas a estos grupos excluidos y explotados constituyen un paso hacia la dura tarea de consolidar una sociedad más justa, que respete los diversos proyectos de vida en dignidad, y que, mirando la realidad, construya un derecho emancipador y transformador.

\section{BIBLIOGRAFÍA}

Bartlett, Katharine. "Métodos jurídicos feministas". En Marisol Fernández y Félix Morales coord., Métodos feministas en el Derecho. Lima: Palestra, 2011.

Bermúdez, Violeta. La regulación jurídica del aborto en América Latina y el Caribe. Estudio comparativo. Lima: CLADEM, 1998.

Bravo, Diego, y Marcel Bonilla. "74 mujeres fueron enjuiciadas por abortar los dos últimos años”. El Comercio, Actualidad, 25 de enero de 2016. Fecha de consulta: 12-01-2017. Disponible en $\measuredangle$ http://www.elcomercio.com/actualidad/ecuador-mujeres-juicios-aborto-carcel. html..

51. Pitch, Un derecho para dos. La construcción jurídica de género, sexo y sexualidad, 99.

52. A manera de ejemplo, en Argentina, país que criminaliza la interrupción de la gestación, se presenta 1 aborto por cada recién nacido. En Suecia, Estado que ha legalizado la interrupción del embarazo, existe 1 aborto por cada 5 recién nacidos. Mario Sebastiani, "El aborto en condiciones de riesgo en un contexto legal restrictivo y según una visión de la Salud Pública”, en Marcela Aszkenazi, comp., Clausuras y aperturas. Debates sobre el aborto (Buenos Aires: Espacio Editorial, 2007), 12. 
Caicedo, Danilo, “El bloque de constitucionalidad en el Ecuador. Derechos humanos más allá de la Constitución". FORO: Revista de Derecho, No. 12, Universidad Andina Simón Bolívar, Sede Ecuador/Corporación Editora Nacional (2009).

Carrara, Francisco. Programa de Derecho criminal, t. III, 3a. ed. Bogotá: Temis, 1991.

Cevallos, María Rosa. El temor encarnado. Quito: FLACSO Ecuador, 2012.

Colectivo Político Luna Creciente. Estado de arte de derechos sexuales y derechos reproductivos de las mujeres en el Ecuador. Quito: Fondo de Cooperación al Desarrollo de Solidaridad Socialista Belga, 2013.

Coomaraswamy, Radhira. "Informe de la Relatoría Especial sobre la violencia contra la mujer, sus causas y consecuencias". Revista Argentina de Derechos Humanos. Buenos Aires (2001).

Coordinadora Política Juvenil por la Equidad de Género. ;De eso no se habla! Quito: CPJ.

De Lama Ayma, Alejandra. Discriminación múltiple I. Barcelona: Universidad Autónoma de Barcelona.

Domingo, Rafael. "El aborto en Alemania: (Observaciones sobre la sentencia del Tribunal Constitucional alemán de 28.5.1993)”. Cuadernos de Bioética (1994).

Espinoza, Henry, y Lizbeth López. “Aborto inseguro en América Latina y el Caribe: definición del problema y su prevención”. Gaceta Médica de México, vol. 139, suplem. 1 (julio-agosto 2003). Disponible en 〈http://www.medigraphic.com/pdfs/gaceta/gm-2003/gms031c.pdf〉.

Facio, Alda. "Metodología para el análisis de género del fenómeno legal”. En Ramiro Ávila Santamaría, Judith Salgado y Lola Valladares, comp., El género en el derecho: Ensayos críticos. Quito: Ministerio de Justicia y Derechos Humanos, 2009.

Faúndes, Aníbal, y José Barzelatto. El drama del aborto. En busca de consensos. Buenos Aires: Paidós, 2011.

Ferrajoli, Luigi. Derechos y garantías: la ley del más débil, 2a. ed. Madrid: Trotta, 1999.

Frejka, Tomas, Lucille Atkin y Olga Toro. Programa de investigación para la prevención del aborto inducido en condiciones riesgosas y sus consecuencias adversas en América Latina y el Caribe. México: The Population Council, 1989.

Friedman, Daniel. Análisis jurídico de la despenalización del aborto en el Ecuador en casos de violación. Tesis de doctorado en Derecho, Facultad de Ciencias Jurídicas y Sociales, Universidad Internacional SEK, Quito, 2005.

Gómez de la Torre, Virginia. "Derechos económicos, sociales y culturales. La salud de las mujeres al 2010 en el Ecuador”. En Informe Derechos Humanos Ecuador 2009-2010. Quito: Centro Regional de Derechos Humanos y Justicia de Género, 2011.

Gómez, Virginia, y Marta López. Decisiones cotidianas. Quito: Fundación Desafío/Hivos, 2011.

Guerra, Elsa. "La constitucionalidad del aborto voluntario en Ecuador". Tesis de maestría, Universidad Andina Simón Bolívar, Sede Ecuador, 2013. 
Inter-American Commission of Women. Informe hemisférico sobre violencia sexual y embarazo infantil en los Estados Parte de la Convención de Belém do Pará. Washington D. C.: OEA, 2016.

Larrea, Sara. Diagnóstico de la situación de la promoción, oferta y demanda de la anticoncepción de emergencia en Loja, Guayas, Pichincha, Esmeraldas y Chimborazo. Quito: Fundación Desafío y Coordinadora Juvenil por la Equidad de Género, 2010.

MacKinnon, Catherine. Hacia una teoría feminista del Estado. Madrid: Cátedra, 1995.

Medellín, Ximena. Principio Pro persona. México: Suprema Corte de Justicia de la Nación/ Oficina en México del Alto Comisionado de las Naciones Unidas para los Derechos Humanos, 2013.

Ministerio de Salud Pública. Plan Nacional de Reducción Acelerada de la Mortalidad Materna y Neonatal. Septiembre de 2008.

Pitch, Tamar. Un derecho para dos. La construcción jurídica de género, sexo, sexualidad. Madrid: Trotta, 2003.

Plan V. "Plan Familia Ecuador o un retroceso de cien años". Plan V, 14 de marzo de 2015.

Salgado, Judith. "Aportes al debate sobre igualdad desde el feminismo”. Revista Aportes Andinos, No. 9. Universidad Andina Simón Bolívar (2004).

---. Manual de formación en género y derechos humanos. Quito: Universidad Andina Simón Bolívar, Sede Ecuador/Corporación Editora Nacional, 2013.

Scott, Joan. "El género una categoría útil para el análisis histórico". En Marta Lamas, comp., El género. La construcción cultural de la diferencia sexual. México: UNAM, 2003.

Sebastiani, Mario. "El aborto en condiciones de riesgo en un contexto legal restrictivo y según una visión de la Salud Pública”. En Marcela Aszkenazi, comp., Clausuras y aperturas. Debates sobre el aborto. Buenos Aires: Espacio Editorial, 2007.

\section{Otros}

Colombia. Corte Constitucional de Colombia, Sentencia C-355 de 2006.

Comisión Interamericana de Derechos Humanos. Audiencia temática: criminalización del aborto en Sudamérica, 15 de marzo de 2006.

Comité de América Latina y el Caribe para la Defensa de los Derechos de la Mujer (CLADEM). Investigación 2009.

Corte Interamericana de Derechos Humanos. Caso Artavia Murillo y Otros ("Fecundación in vitro") vs. Costa Rica, sentencia de 28 de noviembre de 2012.

Ecuador. Código Orgánico Integral Penal. Registro Oficial Suplemento, No. 180, 10 de febrero de 2014.

---. Constitución de la República del Ecuador, Registro Oficial, No. 449, 20 de octubre de 2008.

Encuesta Demográfica y de Salud Materna e Infantil del Ecuador ENDEMAIN. 2004. 
FORO 29, I semestre 2018

Organización de las Naciones Unidas. Comité de la CEDAW. Recomendación No. 28.

---. Dictamen del Comité de Derechos Humanos, No. 11 53/2003, 85 Período de Sesiones, 17 de noviembre de 2005.

Organización Panamericana de la Salud. Salud en las Américas 2007, vol. I, Regional. Washington D. C.: OPS, 2007.

Secretaría Nacional de Planificación y Desarrollo del Ecuador. Plan Nacional del Buen Vivir 2013-2017. Quito: SENPLADES.

Fecha de recepción: 17 de mayo de 2017.

Fecha de aprobación: 14 de septiembre de 2017. 